\title{
NOTE ON BOUNDS FOR DETERMINANTS WITH DOMINANT PRINCIPAL DIAGONAL
}

\author{
A. M. OSTROWSKI
}

1. Let $A=\left|a_{\mu \nu}\right|(\mu, \nu=1, \cdots, n)$ be a determinant and put

$$
\alpha_{\mu \nu}=\left|a_{\mu \nu}\right|(\mu, \nu=1, \cdots, n), \alpha_{\mu \mu} \neq 0(\mu=1, \cdots, n),
$$

$$
\sum_{\nu=1}^{n} \alpha_{\mu \nu}-\alpha_{\mu \mu}=s_{\mu}=\sigma_{\mu} \alpha_{\mu \mu}
$$

Under the hypothesis that all $\sigma_{\mu}$ are less than 1, I proved (1937) ${ }^{1}$ that

$$
|A| \geqq \prod_{\mu=1}^{n}\left(\alpha_{\mu \mu}-s_{\mu}\right)
$$

This inequality provides a simple lower bound for $|A|$ which is however of the wrong order if all $s_{\mu}$ tend to 0 ; for in this case the difference $A-a_{11} a_{22} \cdots a_{n n}$ is at least of the second order in the $\alpha_{\mu \nu}(\mu \neq \nu)$.

2. Some months later' I gave another estimate for $|A|$. Suppose that the $\sigma_{\mu}$ are arranged decreasingly:

$$
\sigma_{m_{1}} \geqq \sigma_{m_{2}} \geqq \cdots \geqq \sigma_{m_{n}},
$$

then we have:

$$
\begin{aligned}
\left(\alpha_{m_{1} m_{1}} \alpha_{m_{2} m_{2}}\right. & \left.+s_{m_{1}} s_{m_{2}}\right)\left(\alpha_{m_{3} m_{3}} \alpha_{m_{4} m_{4}}+s_{m_{3}} s_{m_{4}}\right) \cdots \geqq|A| \\
& \geqq\left(\alpha_{m_{1} m_{1}} \alpha_{m_{2} m_{2}}-s_{m_{1}} s_{m_{2}}\right)\left(\alpha_{m_{3} m_{3}} \alpha_{m_{4} m_{4}}-s_{m_{8}} s_{m_{4}}\right) \cdots,
\end{aligned}
$$

where if $n$ is odd, the last factors to the left and to the right in (4) are resp. $\alpha_{m_{n-2} m_{n-2}} \alpha_{m_{n-1} m_{n-1}} \pm s_{m_{n-2}} s_{m_{n-1}}$. All other couples $\alpha_{m_{v} m_{v}}, s_{m_{v}}$ are paired together as shown in (4).

Received by the editors March 17, 1951.

${ }^{1}$ A. Ostrowski, Sur la determination des bornes inférieures pour une classe des determinants, Bull. Sci. Math. (2) vol. 61 (1937) pp. 19-32. The fact that, if all $\sigma_{\mu}$ are less than $1, A$ does not vanish is due to Hadamard and others.

2 A. Ostrowski, Ueber die Determinanten mit überwiegender Hauptdiagonale, Comment. Math. Helv. vol. 10 (1937) pp. 69-96. I use this opportunity to mention the following misprints in this paper: p. 70, 1. 9, f.a., read $\left|h_{\mu \mu}\right|$ instead of $h_{\mu \mu}$; p. 73, formula (13), read $\left|h_{\mu \mu}\right|$ instead of $h_{\mu \mu}$ and $\sum_{\nu_{-1}, \nu \neq \mu}^{n}$ instead of $\sum_{\mu-1, \mu \neq \nu}^{n} ;$ p. 73, 1.4, f.b., read 1881 instead of 1899 ; p. 76, the right-side product-sign in the formula (18) is to be dropped; p. 86 , in the formula $(11,1)$ read on the left side $m_{\mu \mu} y_{\mu}$ instead of $y_{\mu}$, and on the right side $M$ instead of $1 ;$ p. 96,1 . 7, f.b., read $s_{1} / s_{2}$ instead of $s_{2} / s_{1}$. 
This result gives not only the best order of $A-a_{11} \cdots a_{n n}$, but is also "the best" in this sense, that both bounds can be attained for any given sets of positive $\alpha_{11}, \cdots, \alpha_{n n}$ and of non-negative $s_{1}, \cdots$, $s_{n}$. Besides, for the validity of (4) it is already sufficient that $\sigma_{m_{1}} \sigma_{m_{2}}$ $<1$, while $\sigma_{m_{1}}$ can be greater than 1 .

3. In two recent publications G. B. Price ${ }^{3}$ and R. Oeder ${ }^{4}$ suggested a new approach to the problem in using the expressions

$$
r_{\mu}=\sum_{\nu=\mu+1}^{n} \alpha_{\mu \nu}, \quad l_{\mu}=\sum_{\nu=1}^{\mu-1} \alpha_{\mu \nu} .
$$

G. B. Price showed that (2) can be improved to the inequality

$$
\prod_{\mu=1}^{n}\left(\alpha_{\mu \mu}+r_{\mu}\right) \geqq|A| \geqq \prod_{\mu=1}^{n}\left(\alpha_{\mu \mu}-r_{\mu}\right)
$$

under the assumption that all $\sigma_{\mu}$ are less than 1 , while $R$. Oeder gives, under the assumption that all $\sigma_{\mu}$ are less than or equal to 1 , the inequality

$$
|A| \geqq \alpha_{11} \prod_{\mu=2}^{n}\left(\alpha_{\mu \mu}-l_{\mu}\right) .
$$

However, the very remarkable inequalities (6) and ( $\left.6^{\prime}\right)$ can still be improved in such a way that $A-a_{11} a_{22} \cdots a_{n n}$ becomes of the second order and the $l_{\mu}$ are used together with the $r_{\mu}$. Put

$$
\sigma=\max _{\mu} \sigma_{\mu}
$$

Then we have for an arbitrary index $k$ from $1, \cdots, n$ :

$$
\begin{aligned}
\alpha_{k k} \prod_{\mu=1}^{k-1}\left(\alpha_{\mu \mu}+\sigma r_{\mu}\right) \prod_{\mu=k+1}^{n}\left(\alpha_{\mu \mu}+\sigma l_{\mu}\right) \geqq|A| \\
\geqq \alpha_{k k} \prod_{\mu=1}^{k-1}\left(\alpha_{\mu \mu}-\sigma r_{\mu}\right) \prod_{\mu=k+1}^{n}\left(\alpha_{\mu \mu}-\sigma l_{\mu}\right)
\end{aligned}
$$

under the assumption that all $\sigma_{\mu}$ are less than or equal to 1. In (8), $\sigma$ can be replaced by

$$
t_{\mu}=\max _{\kappa \neq \mu} \sigma_{\kappa}
$$

${ }^{3}$ G. B. Price, Bounds for determinants with dominant principal diagonal, Proceedings of the American Mathematical Society vol. 2 (1951) pp. 497-502.

4. Oeder, Amer. Math. Monthly vol. 58 (1951) p. 37, problem E. 949. 
4. Still, if we wish to obtain the best possible estimates of $|A|$, it is better not to use any of the formulae (8), but to avail oneself repeatedly of the following inequality in which $A_{m}$ is the principal minor of $A$ corresponding to $a_{m m}$ :

$$
\left(\alpha_{m m}+t_{m} s_{m}\right)\left|A_{m}\right| \geqq|A| \geqq\left(\alpha_{m m}-t_{m} s_{m}\right)\left|A_{m}\right| .
$$

This inequality is valid if we have

$$
t_{m} \leqq 1, \quad t_{m} \sigma_{m}<1 .
$$

We have even, under the hypothesis (11), the following a little more general relation

$$
A=A_{m}\left(a_{m m}+\theta_{m} t_{m} s_{m}\right), \quad\left|\theta_{m}\right| \leqq 1 .
$$

To obtain (8) from (10) we use (10) repeatedly in applying it with $m=1$, then in $\left|A_{1}\right|$ with $m=2, \cdots$, and so forth, $k-1$ times and then with $m=n, n-1, \cdots, k+1$.

5. On the other hand, it is obviously not at all necessary or even advisable to use (10) in such a way as to obtain at each step one of the $r_{\mu}$ or $l_{\mu}$, since in any case the number of the $\alpha_{\mu \nu}$ entering into the expressions corresponding to $s_{m}$ is diminished by 1 at each step; we can further combine the use of the columns with that of the rows, as soon as the sums in the columns corresponding to $s_{m}$ have become less than the moduli of the corresponding elements in the principal diagonal.

6. Our proof of the inequality (10) uses a very remarkable inequality valid for the elements of the inverse matrix of $A$, when all $\sigma_{\mu}$ are less than 1 . Put

$$
A^{-1}=B=\left(b_{\mu \nu}\right), \quad\left|b_{\mu \nu}\right|=\beta_{\mu \nu} \quad(\mu, \nu=1, \cdots, n) .
$$

Then we have

$$
\beta_{\mu \nu} \leqq \sigma_{\mu} \beta_{\nu \nu} \quad(\mu \neq \nu),
$$

and further

$$
b_{\mu \mu}=\frac{1}{a_{\mu \mu}+\theta_{\mu} t_{\mu} s_{\mu}}, \quad\left|\theta_{\mu}\right| \leqq 1 .
$$

7. Proof of (13). We have for $\mu \neq \nu$

$$
\sum_{\kappa} a_{\mu k} b_{k \nu}=0 \quad(\mu \neq \nu) .
$$

Let $B_{v}$ be the maximum of $\beta_{k v}$ in the $\nu$ th column: 


$$
\max \beta_{k \nu}=B_{\nu}
$$

Then we have from (15):

$$
\alpha_{\mu \mu} \beta_{\mu \nu}=\left|a_{\mu \mu} b_{\mu \nu}\right|=\left|-\sum_{\kappa \neq \mu} a_{\mu \kappa} b_{\kappa \nu}\right| \leqq B, \sum_{\kappa \neq \mu} \alpha_{\mu \kappa}=\sigma_{\mu} \alpha_{\mu \mu} B_{\nu},
$$

and

$$
\beta_{\mu \nu} \leqq \sigma_{\mu} B_{\nu}<B_{\nu}
$$

But then it follows from (16) and (18) that $B_{\nu}=\beta_{\nu v}$, and (13) is proved.

8. Proof of (14). To prove (14) we start for a fixed $\mu$ from

$$
a_{\mu \mu} b_{\mu \mu}+\sum_{\kappa \neq \mu} a_{\mu \kappa} b_{\kappa \mu}=1 \quad(\mu=1, \cdots, n)
$$

and obtain by using (13) and (9):

$$
\left|a_{\mu \mu} b_{\mu \mu}-1\right| \leqq \sum_{\kappa \neq \mu} \alpha_{\mu \kappa} \beta_{\kappa \mu} \leqq \sum_{\kappa \neq \mu} \alpha_{\mu \kappa} \sigma_{\kappa} \beta_{\mu \mu} \leqq t_{\mu} \beta_{\mu \mu} s_{\mu}
$$

and therefore for a certain $\theta_{\mu}$ with $\left|\theta_{\mu}\right| \leqq 1$ :

$$
a_{\mu \mu} b_{\mu \mu}=1-\theta_{\mu} t_{\mu} s_{\mu} b_{\mu \mu}
$$

(14) follows now at once.

9. Proof of $\left(10^{\prime}\right)$. We assume first that all $\sigma_{\mu}$ are less than 1 . Develop then $A$ in the elements of the $m$ th row; we obtain, denoting the algebraic complement of $a_{\mu \nu}$ by $A_{\mu \nu}$,

$$
A=A_{m}\left(a_{m m}+\sum_{\nu \neq m} a_{m \nu} \frac{A_{m \nu}}{A_{m m}}\right) ;
$$

obviously $A_{m}=A_{m m}$ is not equal to 0 , since Hadamard's theorem can be certainly applied to this principal minor of $A$.

On the other hand we have by (12) and (13):

$$
\frac{A_{m \nu}}{A_{m m}}=\frac{b_{\nu m}}{b_{m m}}, \quad\left|\frac{A_{m \nu}}{A_{m m}}\right|=\frac{\beta_{v m}}{\beta_{m m}} \leqq \sigma_{\nu} \quad(\nu \neq m),
$$

and therefore

$$
\left|\frac{A}{A_{m}}-a_{m m}\right| \leqq \sum_{\nu \neq m} \alpha_{m \nu} \sigma_{\nu} \leqq t_{m} s_{m}
$$

$\left(10^{\prime}\right)$ is now proved, if all $\sigma_{\mu}$ are less than 1 . 
10. It is now easy to see that $\left(10^{\prime}\right)$ is also true if all $\sigma_{\mu}$ are less than or equal to 1 . Indeed, multiply then all elements of $A$ off the principal diagonal by $t, 0<t<1$. We obtain a determinant for which (24) has been already proved and get thus with $t \uparrow 1$ the inequality (24) and thence $\left(10^{\prime}\right)$ under the assumption that all $\sigma_{\mu}$ are $\leqq 1$.

11. Suppose now that the hypothesis (11) is satisfied. We can then assume that in particular

$$
\sigma_{m}>1, \quad t_{m}<1 .
$$

Let then $t$ be such that

$$
t_{m}<t<\frac{1}{\sigma_{m}}<1 .
$$

We multiply the $m$ th row of $A$ by $t$ and divide the $m$ th column by $t$. We obtain a new determinant $A^{\prime}$ and denote the expressions $s_{\mu}, \sigma_{\mu}$, and $t_{m}$ corresponding to $A^{\prime}$ by $s_{\mu}^{\prime}, \sigma_{\mu}^{\prime}$, and $t_{m}^{\prime}$. We have obviously

$$
\begin{aligned}
& \sigma_{m}^{\prime}=t \sigma_{m}<1, \quad s_{m}^{\prime}=t s_{m}, \\
& \sigma_{\mu}^{\prime} \leqq \frac{1}{t} \sigma_{\mu}<1 \\
& t_{m}^{\prime}=\max _{\mu \neq m} \sigma_{\mu}^{\prime} \leqq \frac{t_{m}}{t} .
\end{aligned}
$$

But then we can apply $\left(10^{\prime}\right)$ to $A^{\prime}$ and obtain by (27) and (29):

$$
\frac{A}{A_{m}}-a_{m m}=\theta_{m}^{\prime} t_{m}^{\prime} s_{m}^{\prime}=\theta_{m} t_{m} s_{m},
$$

where $\left|\theta_{m}\right| \leqq\left|\theta_{m}^{\prime}\right| \leqq 1$. The proof of $\left(10^{\prime}\right)$ is now completed.

UNIVERSITY OF BASLE 